www.asianjournalofmycology.org Article

Doi 10.5943/ajom/1/1/7

\title{
Checklist of microfungi on grasses in Thailand (excluding bambusicolous fungi)
}

\author{
Goonasekara ID ${ }^{1,2,3}$, Jayawardene $\mathrm{RS}^{1,2}$, Saichana $\mathrm{N}^{3}$, Hyde $\mathrm{KD}^{1,2,3,4}$ \\ ${ }^{1}$ Center of Excellence in Fungal Research, Mae Fah Luang University, Chiang Rai 57100, Thailand \\ ${ }^{2}$ School of Science, Mae Fah Luang University, Chiang Rai 57100, Thailand \\ ${ }^{3}$ Key Laboratory for Plant Biodiversity and Biogeography of East Asia (KLPB), Kunming Institute of Botany, Chinese \\ Academy of Science, Kunming 650201, Yunnan, China \\ ${ }^{4}$ World Agroforestry Centre, East and Central Asia, 132 Lanhei Road, Kunming 650201, Yunnan, China
}

Goonasekara ID, Jayawardene RS, Saichana N, Hyde KD 2018 - Checklist of microfungi on grasses in Thailand (excluding bambusicolous fungi). Asian Journal of Mycology 1(1), 88-105, Doi 10.5943/ajom/1/1/7

\begin{abstract}
An updated checklist of microfungi, excluding bambusicolous fungi, recorded on grasses from Thailand is provided. The host plant(s) from which the fungi were recorded in Thailand is given. Those species for which molecular data is available is indicated. In total, 172 species and 35 unidentified taxa have been recorded. They belong to the main taxonomic groups Ascomycota: 98 species and 28 unidentified, in 15 orders, 37 families and 68 genera; Basidiomycota: 73 species and 7 unidentified, in 8 orders, 8 families and 18 genera; and Chytridiomycota: one identified species in Physodermatales, Physodermataceae.
\end{abstract}

Key words - Ascomycota - Basidiomycota - Chytridiomycota - Poaceae - molecular data

\section{Introduction}

Grasses constitute the plant family Poaceae (formerly Gramineae), which includes over 10,000 species of herbaceous annuals, biennials or perennial flowering plants commonly known as true grains, pasture grasses, sugar cane and bamboo (Watson 1990, Kellogg 2001, Sharp \& Simon 2002, Encyclopedia of Life 2018). With a worldwide distribution, except in parts of Antarctica and Greenland, grasses cover at least 20\% of the earth's surface (Wheeler et al. 1990, Gibson 2009). They are known to have a Gondwanan origin evolving 80-100 million years ago, or even earlier (Prasad et al. 2011). Grasses play a crucial role in any ecological system as an undergrowth and sometimes as weeds, while natural and sown/planted grasslands are used for grazing by herbivores, the first members of the food cycle (Watson 1990, Kellogg 2001, Sharp \& Simon 2002, Dai et al. 2017, Thambugala et al. 2017). Economically, Poaceae are the most important group of monocots, comprising a variety of plant species with great agricultural value (Encyclopedia of Life 2018, The Plant List 2018). Humans depend on grasses, especially cereal crops such as barley, maize, rice and wheat for food, and as fodder for animals. Grasses are useful in the production of alcoholic beverages, crystal sugar and paper, as an energy source or biofuel, in ornamental landscaping and various construction purposes, to name a few uses (Kellogg 2001, Gibson 2009, Thambugala et al. 2017, Encyclopedia of Life 2018).

Microfungi, or ascomycetes and their asexual morphs can occur on grasses as pathogens, endophytes, epiphytes or saprobes (Wong \& Hyde 2001, Manamgoda et al. 2011, Hyde et al. 2013, 
Quaedvlieg et al. 2013, Liu et al. 2015b, Dai et al. 2017). Most commonly found microfungi are either Dothideomycetes or Sordariomycetes. In many cases the asexual morphs of these fungi cause a wide economic impact as plant pathogens (Manamgoda et al. 2012a, b, 2013, Quaedvlieg et al. 2013, Hyde et al. 2013, 2016, Phookamsak et al. 2014a, Liu et al. 2015b, Thambugala et al. 2017). The fungal pathogens on grasses have been studied by several authors, especially those associated with Zea mays and other cereal crops (Lamprecht et al. 2011, Manamgoda et al. 2012a, 2015, Damm et al. 2014, Bakhshi et al. 2015). Some interesting groups of fungi included in these studies are Alternaria, Bipolaris, Curvularia, and especially the asexual morph genera of the family Phaeosphaeriaceae (Manamgoda et al. 2012a, b, 2015, Phookamsak et al 2014a). The book on smut fungi of the world (Vánky 2012) includes an extensive record of the smut diseases occurring on grasses. Cercosporoid fungi occurring on Poaceae have been researched by Braun et al. (2015). The fungus Pseudopithomyces chartarum is known to cause facial eczema disease in sheep feeding on pasture lands (Brook 1963). Various studies related to fungal endophytes and their effect towards litter decomposition (Purahong \& Hyde 2011), have been conducted in the past years. Fungal endophytic genera Acremonium, Balansia, Claviceps, Epichlö̈, Myriogenospora and Neotyphodium are known to produce alkaloid compounds within their host plant tissues causing cattle-poisoning, ergot poisoning, intoxication, "rye-grass staggers" and narcosis in grazing cattle, horses and sheep, leading to heavy economic losses (Clay 1988). However, the presence of endophytes is considered a defense mechanism against herbivory, as the grasses will be less prone to attack by insects and most cattle will avoid feeding on them (Clay 1988). In addition, these grasses show an enhanced growth, increase in plant biomass, resistance to drought, flood and disease (Clay 1988, Clay et al. 1993, Saikonnen et al. 1999).

More recent studies on the saprobic fungi occurring on various grass hosts, have attempted to introduce or resolve the classification of species belonging to the families Apiosporaceae, Bambusicolaceae, Cainiaceae, Clavicipitaceae, Didymellaceae, Didymosphaeriaceae, Massarinaceae, Mycosphaerellaceae, Periconiaceae, Phaeosphaeriaceae, Pleosporaceae, Roussoellaceae and Sporormiaceae (Liu et al. 2015b, Li et al. 2016, Hyde et al. 2016, Maharachchikumbura et al. 2016, Wijayawardene et al. 2016, Thambugala et al. 2017). These studies indicate that they have a great diversity, however, there is a lack of a thorough and collective inventory for Thailand or the South East Asian region. For many species, there is a lack of molecular analysis (Bhilabutra et al. 2010, Hyde et al. 2013, Thambugala et al. 2017). Therefore, there is a need for re-collection of microfungi on grasses and a reassessment of their taxonomic placements, enabled by both morphological and molecular analyses. This checklist aims to provide a complete as possible list of microfungi recorded from grasses in Thailand (excluding those recorded on bamboo), with their host plants and information as to whether molecular data are available in GenBank. It will be a useful guide for the study of microfungi and their host distribution in Thailand.

\section{Materials \& Methods}

The checklist is based on published articles in journals, books, web-based resources such as reports on host plants, USDA database (Farr \& Rossman 2018), The Smut fungi of Thailand and Food and Agriculture Organization of the United Nations (FAO) websites, and graduate student theses. The checklist (Table 1) includes fungal species names, families and host species names or family. The fungal classification follows Wijayawardene et al. (2018). The names are used according to Index Fungorum (2018) and Wijayawardene et al. (2018). The host name given in the original citation is sometimes changed to be consistent with current taxonomy based on The Plant List (http://www.theplantlist.org). Fungal genera and species are listed in alphabetical order. Species for which molecular data is available in GenBank are marked with an asterisk $(*)$; it should be noted that among them some were introduced from hosts other than grasses or based only on morphology. In a few cases, neither the species nor a proper synonym was identified, therefore the species name is used as originally cited. 


\section{Results}

Table 1 Checklist of microfungi on grasses (excluding bambusicolous fungi) in Thailand.

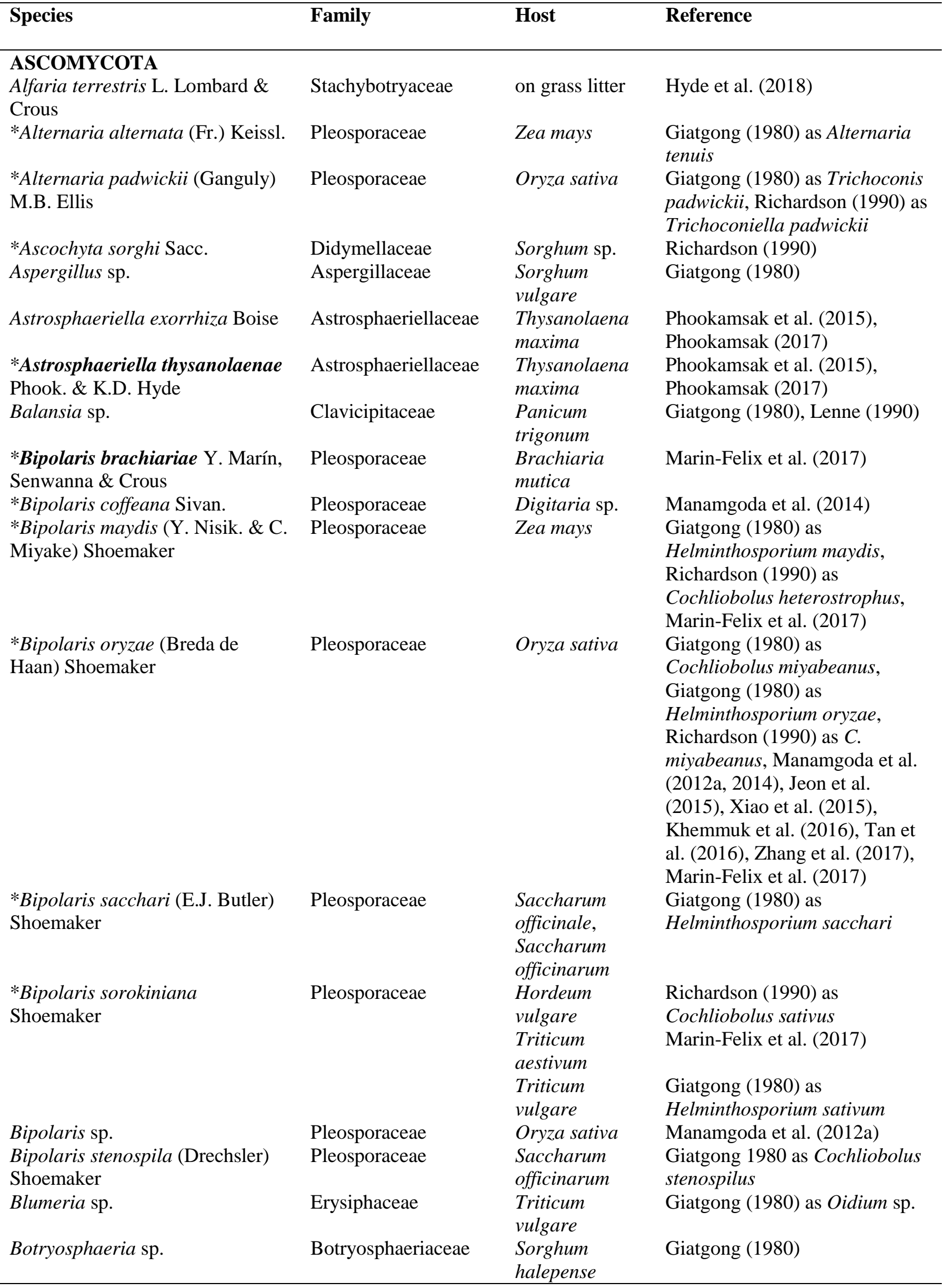


Table 1 Continued.

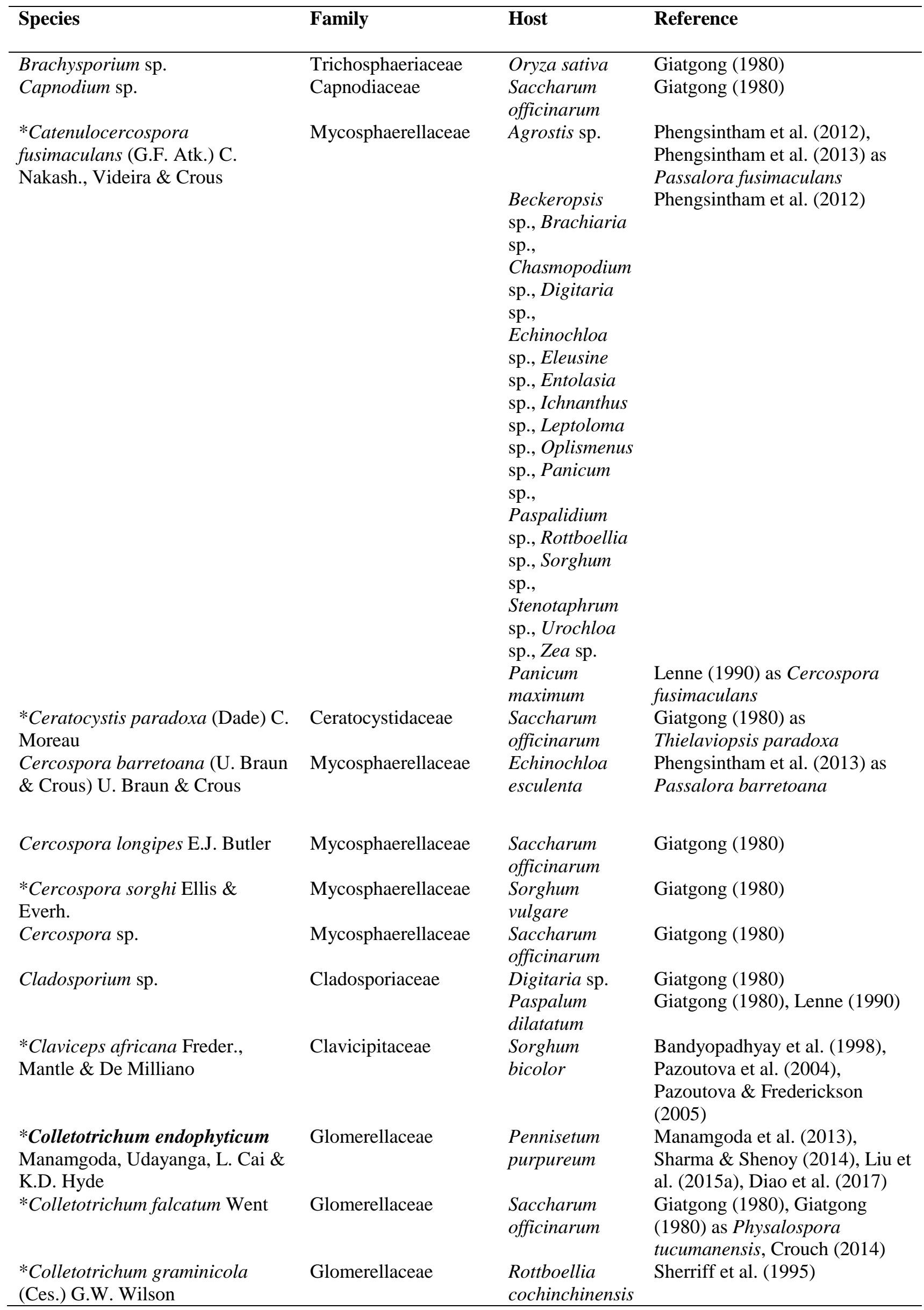


Table 1 Continued.

\begin{tabular}{|c|c|c|c|}
\hline Species & Family & Host & Reference \\
\hline & & $\begin{array}{l}\text { Sorghum } \\
\text { sudanense, } \\
\text { Sorghum } \\
\text { vulgare, Zea } \\
\text { mays }\end{array}$ & Giatgong (1980) \\
\hline $\begin{array}{l}\text { *Colletotrichum siamense } \\
\text { Prihast., L. Cai \& K.D. Hyde }\end{array}$ & Glomerellaceae & $\begin{array}{l}\text { Cymbopogon } \\
\text { citratus }\end{array}$ & Munir et al. (2016) \\
\hline Colletotrichum sp. & Glomerellaceae & $\begin{array}{l}\text { Digitaria sp., } \\
\text { Sorghum } \\
\text { vulgare }\end{array}$ & Giatgong (1980) \\
\hline $\begin{array}{l}\text { *Coniothyrium chiangmaiense } \\
\text { Goonas., Thambug. \& K.D. Hyde }\end{array}$ & Coniothyriaceae & on grass litter & Thambugala et al. (2017) \\
\hline *Curvularia alcornii & \multirow[t]{4}{*}{ Pleosporaceae } & Panicum sp. & Manamgoda et al. (2012a, b) \\
\hline Manamgoda, L. Cai \& K.D. Hyde & & $\begin{array}{l}\text { Pennisetum } \\
\text { clandestinum }\end{array}$ & Manamgoda et al. (2012a) \\
\hline & & Zea mays & $\begin{array}{l}\text { Manamgoda et al. (2012a, b), } \\
\text { Khemmuk et al. (2016) }\end{array}$ \\
\hline & & Zea sp. & Manamgoda et al. (2015) \\
\hline \multirow[t]{2}{*}{$\begin{array}{l}\text { *Curvularia asiatica } \\
\text { Manamgoda, L. Cai \& K.D. Hyde }\end{array}$} & \multirow[t]{2}{*}{ Pleosporaceae } & $\begin{array}{l}\text { Oryza sativa, } \\
\text { Saccharum } \\
\text { officinarum }\end{array}$ & $\begin{array}{l}\text { Manamgoda et al. (2012a, b, } \\
\text { 2015) }\end{array}$ \\
\hline & & Panicum sp. & $\begin{array}{l}\text { Manamgoda et al. (2012a, b, } \\
\text { 2015), Jeon et al. (2015), } \\
\text { Khemmuk et al. (2016), Zhang } \\
\text { et al. (2018) }\end{array}$ \\
\hline \multirow{3}{*}{$\begin{array}{l}\text { *Curvularia chiangmaiensis Y. } \\
\text { Marín, Senwanna \& Crous } \\
\text { *Curvularia dactyloctenicola Y. } \\
\text { Marín, Senwanna \& Crous } \\
\text { *Curvularia hawaiiensis } \\
\text { (Bugnic. ex M.B. Ellis) }\end{array}$} & Pleosporaceae & Zea mays & Marin-Felix et al. (2017) \\
\hline & Pleosporaceae & $\begin{array}{l}\text { Dactyloctenium } \\
\text { aegyptium }\end{array}$ & Marin-Felix et al. (2017) \\
\hline & Pleosporaceae & Panicum sp. & Manamgoda et al. (2012a, 2015) \\
\hline $\begin{array}{l}\text { Manamgoda, L. Cai \& K.D. Hyde } \\
\text { * Curvularia hominis K.C. Cunha, } \\
\text { Madrid, Gené \& Cano }\end{array}$ & Pleosporaceae & $\begin{array}{l}\text { Echinochloa } \\
\text { sp., Oryza } \\
\text { sativa }\end{array}$ & Manamgoda et al. (2015) \\
\hline \multirow[t]{4}{*}{$\begin{array}{l}\text { *Curvularia lunata (Wakker) } \\
\text { Boedijn }\end{array}$} & \multirow[t]{4}{*}{ Pleosporaceae } & Oryza sativa & $\begin{array}{l}\text { Giatgong (1980), Richardson } \\
\text { (1990) as Cochliobolus lunatus, } \\
\text { Manamgoda et al. (2012a), Xiao } \\
\text { et al.(2015) }\end{array}$ \\
\hline & & Panicum sp. & Manamgoda et al. (2012) \\
\hline & & $\begin{array}{l}\text { Sorghum } \\
\text { vulgare }\end{array}$ & Giatgong (1980) \\
\hline & & Zea mays & $\begin{array}{l}\text { Giatgong (1980), Ariyawansa et } \\
\text { al. (2015a), Manamgoda et al. } \\
\text { (2015) }\end{array}$ \\
\hline \multirow[t]{3}{*}{$\begin{array}{l}\text { *Curvularia nodosa Y. Marín, } \\
\text { Cheew. \& Crous }\end{array}$} & \multirow[t]{3}{*}{ Pleosporaceae } & $\begin{array}{l}\text { Brachiaria } \\
\text { reptans }\end{array}$ & Marin-Felix et al. (2017) \\
\hline & & $\begin{array}{l}\text { Chloris } \\
\text { barbata }\end{array}$ & Marin-Felix et al. (2017) \\
\hline & & $\begin{array}{l}\text { Digitaria } \\
\text { ciliaris }\end{array}$ & Marin-Felix et al. (2017) \\
\hline *Curvularia pallescens Boedijn & Pleosporaceae & Oryza sativa & $\begin{array}{l}\text { Sivanesan (1987) as } \\
\text { Cochliobolus pallescens }\end{array}$ \\
\hline $\begin{array}{l}\text { *Curvularia pseudobrachyspora } \\
\text { Y. Marín, Cheew. \& Crous }\end{array}$ & Pleosporaceae & Eleusine indica & Marin-Felix et al. (2017) \\
\hline
\end{tabular}


Table 1 Continued.

\begin{tabular}{|c|c|c|c|}
\hline Species & Family & Host & Reference \\
\hline Curvularia sp. & Pleosporaceae & $\begin{array}{l}\text { Oryza sativa } \\
\text { Oryza sp., } \\
\text { Sorghum sp. } \\
\text { Sorghum } \\
\text { vulgare, Zea } \\
\text { mays }\end{array}$ & $\begin{array}{l}\text { Manamgoda et al. (2012a, 2015) } \\
\text { Richardson (1990) }\end{array}$ \\
\hline \multirow[t]{3}{*}{$\begin{array}{l}\text { *Curvularia variabilis Y. Marín, } \\
\text { Senwanna \& Crous }\end{array}$} & Pleosporaceae & $\begin{array}{l}\text { Chloris } \\
\text { barbata }\end{array}$ & Marin-Felix et al. (2017) \\
\hline & & $\begin{array}{l}\text { Digitaria } \\
\text { ciliaris }\end{array}$ & Marin-Felix et al. (2017) \\
\hline & & $\begin{array}{l}\text { Imperata } \\
\text { cylindrica }\end{array}$ & Marin-Felix et al. (2017) \\
\hline \multirow[t]{3}{*}{$\begin{array}{l}\text { *Curvularia verruculosa Tandon } \\
\& \text { Bilgrami ex M.B. Ellis }\end{array}$} & Pleosporaceae & $\begin{array}{l}\text { Cynodon } \\
\text { dactylon }\end{array}$ & Marin-Felix et al. (2017) \\
\hline & & Eleusine indica & Marin-Felix et al. (2017) \\
\hline & & $\begin{array}{l}\text { Oryza sativa, } \\
\text { Zea sp. }\end{array}$ & Manamgoda et al. (2015) \\
\hline *Cytospora sacchari E.J. Butler & Cytosporaceae & $\begin{array}{l}\text { Saccharum } \\
\text { officinarum }\end{array}$ & Giatgong (1980) \\
\hline Diaporthe oryzae-sativae Punith. & Diaporthaceae & Oryza sativa & $\begin{array}{l}\text { Punithalingam \& Sharma (1979) } \\
\text { as Ascochyta oryzae, Giatgong } \\
\text { (1980) as A. oryzae, Udayanga } \\
\text { et al. } 2011 \text { as A. oryzae }\end{array}$ \\
\hline Diaporthe sorghicola Punith. & Diaporthaceae & $\begin{array}{l}\text { Sorghum } \\
\text { vulgare }\end{array}$ & $\begin{array}{l}\text { Punithalingam (1975) as } \\
\text { Phomopsis sorghicola }\end{array}$ \\
\hline $\begin{array}{l}\text { *Didymella poaceicola Thambug. } \\
\text { \& K.D. Hyde }\end{array}$ & Didymellaceae & Poaceae & Thambugala et al. (2017) \\
\hline Diplocarpon sp. & Drepanopezizaceae & $\begin{array}{l}\text { Saccharum } \\
\text { officinarum }\end{array}$ & $\begin{array}{l}\text { Giatgong (1980) as } \\
\text { Gloeosporium sp. }\end{array}$ \\
\hline Diplodia sp. & Botryosphaeriaceae & Zea mays & $\begin{array}{l}\text { Giatgong (1980), Richardson } \\
\text { (1990) as Botryodiplodia sp. }\end{array}$ \\
\hline \multicolumn{4}{|l|}{ Thambug. \& K.D. Hyde } \\
\hline $\begin{array}{l}\text { *Epicoccum sorghinum (Sacc.) } \\
\text { Aveskamp, Gruyter \& Verkley }\end{array}$ & Leptosphaeriaceae & $\begin{array}{l}\text { Saccharum } \\
\text { officinale }\end{array}$ & $\begin{array}{l}\text { Giatgong (1980) as } \\
\text { Leptosphaeria sacchari }\end{array}$ \\
\hline $\begin{array}{l}\text { *Epicoccum thailandicum } \\
\text { Goonas., Thambug. \& K.D. Hyde }\end{array}$ & Didymellaceae & on grass litter & Thambugala et al. (2017) \\
\hline $\begin{array}{l}\text { *Exserohilum rostratum } \\
\text { (Drechsler) K.J. Leonard \& Suggs }\end{array}$ & Pleosporaceae & Zea mays & $\begin{array}{l}\text { Richardson }(1990) \text { as } \\
\text { Drechslera halodes }\end{array}$ \\
\hline $\begin{array}{l}\text { *Exserohilum turcicum (Pass.) } \\
\text { K.J. Leonard \& Suggs }\end{array}$ & Pleosporaceae & $\begin{array}{l}\text { Sorghum } \\
\text { vulgare, Zea } \\
\text { mays }\end{array}$ & $\begin{array}{l}\text { Giatgong (1980) as } \\
\text { Helminthosporium turcicum }\end{array}$ \\
\hline Fumago sp. & $\begin{array}{l}\text { Pezizomycotina, } \\
\text { Incertae sedis }\end{array}$ & $\begin{array}{l}\text { Saccharum } \\
\text { officinarum }\end{array}$ & Giatgong (1980) \\
\hline \multirow[t]{2}{*}{ *Fusarium fujikuroi Nirenberg } & Nectriaceae & $\begin{array}{l}\text { Hordeum } \\
\text { vulgare } \\
\text { Oryza sativa }\end{array}$ & $\begin{array}{l}\text { Richardson (1990) as Fusarium } \\
\text { moniliforme } \\
\text { Ou (1985) as Gibberella } \\
\text { fujikuroi, Giatgong (1980) as } G . \\
\text { fujikuroi }\end{array}$ \\
\hline & & $\begin{array}{l}\text { Saccharum } \\
\text { officinarum } \\
\text { Zea mays }\end{array}$ & $\begin{array}{l}\text { Giatgong }(1980) \text { as } F \text {. } \\
\text { moniliforme, G. fujikuroi } \\
\text { Giatgong }(1980) \text { as } F \text {. } \\
\text { moniliforme }\end{array}$ \\
\hline $\begin{array}{l}\text { *Fusarium incarnatum (Desm.) } \\
\text { Sacc. }\end{array}$ & Nectriaceae & Oryza sativa & $\begin{array}{l}\text { Giatgong (1980) as Fusarium } \\
\text { semitectum }\end{array}$ \\
\hline
\end{tabular}


Table 1 Continued.

\begin{tabular}{|c|c|c|c|}
\hline Species & Family & Host & Reference \\
\hline *Fusarium oxysporum Schltdl. & Nectriaceae & Zea mays & $\begin{array}{l}\text { Giatgong (1980) as Fusarium } \\
\text { aurantiacum }\end{array}$ \\
\hline $\begin{array}{l}\text { *Fusarium pallidoroseum } \\
\text { (Cooke) Sacc. }\end{array}$ & Nectriaceae & $\begin{array}{l}\text { Hordeum } \\
\text { vulgare }\end{array}$ & Richardson (1990) \\
\hline *Fusarium solani (Mart.) Sacc. & Nectriaceae & $\begin{array}{l}\text { Hordeum } \\
\text { vulgare }\end{array}$ & Richardson (1990) \\
\hline Fusarium sp. & Nectriaceae & $\begin{array}{l}\text { Oryza sativa } \\
\text { Sorghum sp. } \\
\text { Sorghum } \\
\text { vulgare } \\
\text { Zea mays }\end{array}$ & $\begin{array}{l}\text { Giatgong (1980), Richardson } \\
\text { (1990) } \\
\text { Richardson (1990) } \\
\text { Giatgong (1980) } \\
\text { Giatgong (1980), Giatgong } \\
\text { (1980) as Gibberella sp. }\end{array}$ \\
\hline Gloeocercospora sp. & $\begin{array}{l}\text { Xylariales, Incertae } \\
\text { sedis }\end{array}$ & $\begin{array}{l}\text { Sorghum } \\
\text { vulgare }\end{array}$ & Giatgong (1980) \\
\hline $\begin{array}{l}\text { *Graminopassalora graminis } \\
\text { (Fuckel) U. Braun, C. Nakash., } \\
\text { Videira \& Crous }\end{array}$ & Mycosphaerellaceae & 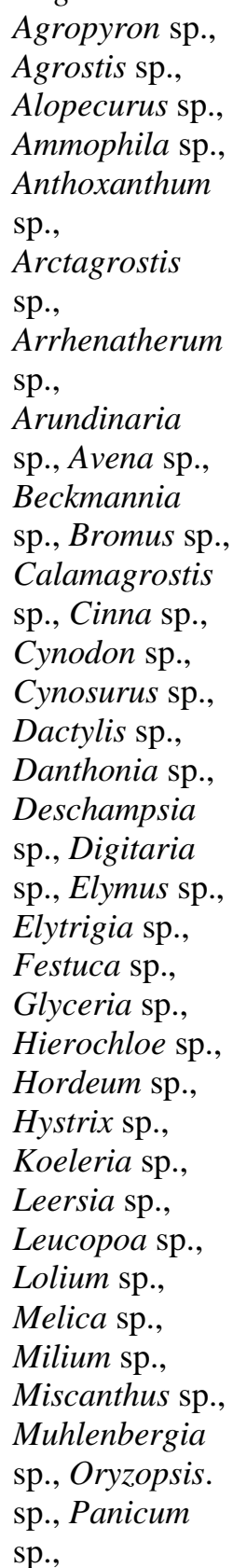 & $\begin{array}{l}\text { Phengsintham et al. (2012) as } \\
\text { Passalora graminis }\end{array}$ \\
\hline
\end{tabular}


Table 1 Continued.

\begin{tabular}{|c|c|c|c|}
\hline Species & Family & Host & Reference \\
\hline & & $\begin{array}{l}\text { Pennisetum sp., } \\
\text { Phleum sp., } \\
\text { Phragmites sp., } \\
\text { Poa sp., } \\
\text { Puccinellia sp., } \\
\text { Roegneria sp., } \\
\text { Secale sp., } \\
\text { Sitanion sp., } \\
\text { Stenotaphrum } \\
\text { sp., Stipa } \mathrm{sp} ., \\
\text { Trisetum sp., } \\
\text { Zea } \text { sp. }\end{array}$ & \\
\hline Helminthosporium sp. & Massarinaceae & $\begin{array}{l}\text { Setaria italic } \\
\text { Zea mays }\end{array}$ & $\begin{array}{l}\text { Lenne }(1990) \\
\text { Giatgong }(1980)\end{array}$ \\
\hline $\begin{array}{l}\text { *Leptosphaerulina saccharicola } \\
\text { Phook., Jian K. Liu \& K.D. Hyde }\end{array}$ & Didymellaceae & $\begin{array}{l}\text { Saccharum } \\
\text { officinarum }\end{array}$ & Phookamsak (2017) \\
\hline $\begin{array}{l}\text { *Macrophomina phaseolina } \\
\text { (Tassi) Goid. }\end{array}$ & Botryosphaeriaceae & $\begin{array}{l}\text { Sorghum } \\
\text { vulgare }\end{array}$ & $\begin{array}{l}\text { Giatgong (1980) as } \\
\text { Botryodiplodia phaseoli }\end{array}$ \\
\hline $\begin{array}{l}\text { *Microdochium albescens } \\
\text { (Thüm.) Hern.-Restr. \& Crous }\end{array}$ & Microdochiaceae & Oryza sativa & $\begin{array}{l}\text { Giatgong (1980) as } \\
\text { Rhynchosporium oryzae }\end{array}$ \\
\hline $\begin{array}{l}\text { *Multiseptospora thailandica } \\
\text { Phookamsak \& K.D. Hyde }\end{array}$ & Parabambusicolaceae & $\begin{array}{l}\text { Thysanolaena } \\
\text { maxima }\end{array}$ & Liu et al. (2015b) \\
\hline $\begin{array}{l}\text { *Nakataea oryzae (Catt.) J. Luo } \\
\& \text { N. Zhang }\end{array}$ & Magnaporthaceae & Oryza sativa & $\begin{array}{l}\text { Giatgong }(1980) \text { as } \\
\text { Leptosphaeria salvinii }\end{array}$ \\
\hline $\begin{array}{l}\text { *Neosetophoma poaceicola } \\
\text { Goonas., Thambug. \& K.D. Hyde }\end{array}$ & Phaeosphaeriaceae & on grass litter & Thambugala et al. (2017) \\
\hline $\begin{array}{l}\text { *Neosphaerellopsis thailandica } \\
\text { Crous \& Trakun. }\end{array}$ & Phaeosphaeriaceae & $\begin{array}{l}\text { Bothriochloa } \\
\text { bladhii }\end{array}$ & Trakunyingcharoen et al. (2014) \\
\hline $\begin{array}{l}\text { *Nigrocornus scleroticus (Pat.) } \\
\text { Ryley }\end{array}$ & Clavicipitaceae & $\begin{array}{l}\text { Cymbopogon } \\
\text { nardus }\end{array}$ & $\begin{array}{l}\text { Ryley (2006) as Balansia } \\
\text { sclerotica }\end{array}$ \\
\hline $\begin{array}{l}\text { *Nigrospora oryzae (Berk. \& } \\
\text { Broome) Petch }\end{array}$ & $\begin{array}{l}\text { Sordariomycetes, } \\
\text { incertae sedis }\end{array}$ & Zea mays & $\begin{array}{l}\text { Richardson (1990) as Khuskia } \\
\text { oryzae }\end{array}$ \\
\hline $\begin{array}{l}\text { *Ophiosphaerella agrostidis } \\
\text { Dern., M.P.S. Câmara, N.R. } \\
\text { O'Neill, Berkum \& M.E. Palm }\end{array}$ & Phaeosphaeriaceae & $\begin{array}{l}\text { on grass litter, } \\
\text { Thysanolaena } \\
\text { maxima }\end{array}$ & $\begin{array}{l}\text { Phookamsak et al. (2014b), } \\
\text { Thambugala et al. (2017) }\end{array}$ \\
\hline Ophiosphaerella-like & $\begin{array}{l}\text { Pleosporales, Incertae } \\
\text { sedis }\end{array}$ & $\begin{array}{l}\text { Imperata } \\
\text { cylindrica }\end{array}$ & Phookamsak (2017) \\
\hline $\begin{array}{l}\text { *Paraconiothyrium } \\
\text { thysanolaenae Phookamsak, } \\
\text { Chethana \& K.D. Hyde }\end{array}$ & Didymosphaeriaceae & $\begin{array}{l}\text { Thysanolaena } \\
\text { maxima }\end{array}$ & Liu et al. (2015b) \\
\hline $\begin{array}{l}\text { *Paraphaeosphaeria graminicola } \\
\text { Thambug. \& K.D. Hyde }\end{array}$ & Didymosphaeriaceae & Poaceae & Thambugala et al. (2017) \\
\hline $\begin{array}{l}\text { Passalora agrostidicola Phengs. } \\
\& \text { U. Braun }\end{array}$ & Mycosphaerellaceae & Agrostis sp. & Braun et al. (2015) \\
\hline $\begin{array}{l}\text { Passalora koepkei (W. Krüger) U. } \\
\text { Braun \& Crous }\end{array}$ & Mycosphaerellaceae & $\begin{array}{l}\text { Saccharum } \\
\text { officinarum }\end{array}$ & $\begin{array}{l}\text { Giatgong (1980) as Cercospora } \\
\text { koepkei }\end{array}$ \\
\hline $\begin{array}{l}\text { *Passalora vaginae (W. Krüger) } \\
\text { U. Braun \& Crous }\end{array}$ & Mycosphaerellaceae & $\begin{array}{l}\text { Saccharum } \\
\text { officinarum }\end{array}$ & $\begin{array}{l}\text { Giatgong (1980) as Cercospora } \\
\text { vaginae }\end{array}$ \\
\hline Pestalotia andropogonis Rostr. & Sporocadaceae & $\begin{array}{l}\text { Andropogon } \\
\text { sorghum }\end{array}$ & Nag Raj (1993) \\
\hline Pestalotia sp. & Sporocadaceae & $\begin{array}{l}\text { Sorghum } \\
\text { vulgare }\end{array}$ & Giatgong (1980) \\
\hline $\begin{array}{l}\text { *Periconia cortaderiae Thambug. } \\
\text { \& K.D. Hyde }\end{array}$ & Periconiaceae & Cortaderia sp. & Thambugala et al. (2017) \\
\hline
\end{tabular}


Table 1 Continued.

\begin{tabular}{|c|c|c|c|}
\hline Species & Family & Host & Reference \\
\hline $\begin{array}{l}\text { *Phaeocytostroma sacchari (Ellis } \\
\text { \& Everh.) B. Sutton }\end{array}$ & Diaporthaceae & $\begin{array}{l}\text { Saccharum } \\
\text { officinarum }\end{array}$ & $\begin{array}{l}\text { Giatgong (1980) as } \\
\text { Melanconium sacchari }\end{array}$ \\
\hline $\begin{array}{l}\text { *Phaeosphaeria chiangraina } \\
\text { Phookamsak \& K.D. Hyde }\end{array}$ & Phaeosphaeriaceae & Oryza sativa & Phookamsak et al. (2014b) \\
\hline $\begin{array}{l}\text { *Phaeosphaeria } \\
\text { thysanolaenicola Phookamsak \& } \\
\text { K.D. Hyde }\end{array}$ & Phaeosphaeriaceae & $\begin{array}{l}\text { Thysanolaena } \\
\text { maxima }\end{array}$ & Phookamsak et al. (2014b) \\
\hline *Phoma insidiosa Tassi & Didymellaceae & $\begin{array}{l}\text { Sorghum } \\
\text { vulgare }\end{array}$ & Giatgong (1980) \\
\hline Phoma sp. & Didymellaceae & $\begin{array}{l}\text { Hordeum } \\
\text { vulgare }\end{array}$ & Richardson (1990) \\
\hline $\begin{array}{l}\text { *Phyllachora chrysopogonicola } \\
\text { Tamakaew, Cheew. \& K.D. Hyde }\end{array}$ & Phyllachoraceae & $\begin{array}{l}\text { Phyllachora } \\
\text { chrysopogonico } \\
\text { la }\end{array}$ & Tamakaew et al. (2017) \\
\hline Phyllachora cynodontis Niessl & Phyllachoraceae & Cynodon sp. & Lenne (1990) \\
\hline Phyllachora sacchari Henn. & Phyllachoraceae & $\begin{array}{l}\text { Sorghum } \\
\text { vulgare }\end{array}$ & $\begin{array}{l}\text { Giatgong (1980) as Phyllachora } \\
\text { sorghi }\end{array}$ \\
\hline Phyllachora sp. & Phyllachoraceae & $\begin{array}{l}\text { Cynodon } \\
\text { dactylon }\end{array}$ & Lenne (1990) \\
\hline $\begin{array}{l}\text { *Phyllachora thysanolaenae } \\
\text { Tamakaew, Maharachch., K.D. } \\
\text { Hyde \& Cheew. }\end{array}$ & Phyllachoraceae & $\begin{array}{l}\text { Thysanolaena } \\
\text { maxima }\end{array}$ & Tamakaew et al. (2017) \\
\hline * Phyllosticta capitalensis Henn. & Phyllostictaceae & $\begin{array}{l}\text { Saccharum } \\
\text { officinarum }\end{array}$ & Wikee et al (2013a, b) \\
\hline Phyllosticta sp. & Phyllostictaceae & Oryza sativa & Giatgong (1980) \\
\hline $\begin{array}{l}\text { *Poaceascoma helicoides Phook. } \\
\text { \& K.D. Hyde }\end{array}$ & Lentitheciaceae & $\begin{array}{l}\text { Digitaria } \\
\text { sanguinalis }\end{array}$ & Phookamsak (2017) \\
\hline $\begin{array}{l}\text { Pseudoacrodictys dimorphospora } \\
\text { Somrith. \& E.B.G. Jones }\end{array}$ & $\begin{array}{l}\text { Ascomycota, incertae } \\
\text { sedis }\end{array}$ & $\begin{array}{l}\text { Arundinaria } \\
\text { pusilla }\end{array}$ & Somrithipol \& Jones (2003) \\
\hline $\begin{array}{l}\text { *Pseudopyricularia bothriochloae } \\
\text { (Crous \& Cheew.) Y. Marín \& } \\
\text { Crous }\end{array}$ & Pyriculariaceae & $\begin{array}{l}\text { Bothriochloa } \\
\text { bladhii }\end{array}$ & $\begin{array}{l}\text { Crous et al. (2013) as } \\
\text { Pyricularia bothriochloae }\end{array}$ \\
\hline $\begin{array}{l}\text { *Pseudotrichia rubriostiolata } \\
\text { (Phook. \& K.D. Hyde) Q. Tian \& } \\
\text { K.D. Hyde }\end{array}$ & Pleomassariaceae & $\begin{array}{l}\text { Thysanolaena } \\
\text { maxima }\end{array}$ & Liu et al. (2015b) \\
\hline $\begin{array}{l}\text { *Pseudotrichia thailandica } \\
\text { (Phook. \& K.D. Hyde) Q. Tian \& }\end{array}$ & Pleomassariaceae & $\begin{array}{l}\text { Thysanolaena } \\
\text { maxima }\end{array}$ & Liu et al. (2015b) \\
\hline K.D. Hyde & & on grass litter & Phookamsak (2017) \\
\hline $\begin{array}{l}\text { *Psiloglonium colihuae (Lorenzo } \\
\text { \& Messuti) E. Boehm }\end{array}$ & Hysteriaceae & $\begin{array}{l}\text { Thysanolaena } \\
\text { maxima }\end{array}$ & Liu et al. (2015b) \\
\hline $\begin{array}{l}\text { *Psiloglonium multiseptatum } \\
\text { Phookamsak \& K.D. Hyde }\end{array}$ & Hysteriaceae & $\begin{array}{l}\text { Thysanolaena } \\
\text { maxima }\end{array}$ & Liu et al. (2015b) \\
\hline Pyrenophora sp. & Pleosporaceae & $\begin{array}{l}\text { Ophiuros } \\
\text { exaltatus }\end{array}$ & Lenne (1990) as Drechslera sp. \\
\hline & & $\begin{array}{l}\text { Oryza sativa, } \\
\text { Sorghum } \mathrm{sp} .\end{array}$ & $\begin{array}{l}\text { Richardson }(1990) \text { as } \\
\text { Drechslera sp. }\end{array}$ \\
\hline $\begin{array}{l}\text { *Pyrenophora tritici-repentis } \\
\text { (Died.) Drechsler }\end{array}$ & Pleosporaceae & $\begin{array}{l}\text { Triticum } \\
\text { vulgare }\end{array}$ & $\begin{array}{l}\text { Giatgong }(1980) \text { as } \\
\text { Helminthosporium tritici- } \\
\text { vulgaris }\end{array}$ \\
\hline * Pyricularia oryzae Cavara & Pyriculariaceae & Oryza sativa & Giatgong (1980) \\
\hline Pyricularia sp. & Pyriculariaceae & $\begin{array}{l}\text { Brachiaria } \\
\text { mutica, } \\
\text { Digitaria sp., } \\
\text { Echinochloa } \\
\text { colonum }\end{array}$ & Giatgong (1980), Lenne (1990) \\
\hline
\end{tabular}


Table 1 Continued.

\begin{tabular}{|c|c|c|c|}
\hline Species & Family & Host & Reference \\
\hline $\begin{array}{l}\text { *Sarocladium oryzae (Sawada) } \\
\text { W. Gams \& D. Hawksw. }\end{array}$ & $\begin{array}{l}\text { Hypocreales, Incertae } \\
\text { sedis }\end{array}$ & $\begin{array}{l}\text { Eragrostis sp. } \\
\text { Ischaemum } \\
\text { rugosum, } \\
\text { Leersia } \\
\text { hexandra } \\
\text { Panicum } \\
\text { repens } \\
\text { Triticum } \\
\text { vulgare } \\
\text { Zoysia matrella } \\
\text { Oryza sativa }\end{array}$ & $\begin{array}{l}\text { Giatgong (1980), Lenne (1990) } \\
\text { Giatgong (1980) } \\
\text { Giatgong (1980) } \\
\text { Giatgong (1980) as } \\
\text { Acrocylindrium oryzae, } \\
\text { Richardson (1990) }\end{array}$ \\
\hline $\begin{array}{l}\text { *Sarocladium strictum }(\mathrm{W} . \\
\text { Gams) Summerb. }\end{array}$ & $\begin{array}{l}\text { Hypocreales, Incertae } \\
\text { sedis }\end{array}$ & $\begin{array}{l}\text { Sorghum } \\
\text { vulgare, Zea } \\
\text { mays }\end{array}$ & $\begin{array}{l}\text { Giatgong (1980) as } \\
\text { Cephalosporium acremonium }\end{array}$ \\
\hline Sclerotium sp. & Sclerotiniaceae & $\begin{array}{l}\text { Saccharum } \\
\text { officinarum }\end{array}$ & Giatgong (1980) \\
\hline $\begin{array}{l}\text { *Setophoma poaceicola Goonas., } \\
\text { Thambug. \& K.D. Hyde }\end{array}$ & Phaeosphaeriaceae & on grass litter & Thambugala et al. (2017) \\
\hline $\begin{array}{l}\text { * Setophoma sacchari (Bitanc.) } \\
\text { Gruyter, Aveskamp \& Verkley }\end{array}$ & Phaeosphaeriaceae & $\begin{array}{l}\text { Saccharum } \\
\text { officinarum }\end{array}$ & $\begin{array}{l}\text { Phookamsak et al. (2014a), } \\
\text { Phookamsak (2017) }\end{array}$ \\
\hline $\begin{array}{l}\text { *Spegazzinia neosundara } \\
\text { Thambug. \& K.D. Hyde }\end{array}$ & Apiosporaceae & Cortaderia sp. & Thambugala et al. (2017) \\
\hline *Sphaerulina oryzina Hara & Mycosphaerellaceae & Oryza sativa & $\begin{array}{l}\text { Richardson }(1990) \text { as } \\
\text { Cercospora oryzae }\end{array}$ \\
\hline $\begin{array}{l}\text { *Stagonospora imperaticola } \\
\text { Phukhams., Thambug. \& K.D. } \\
\text { Hyde }\end{array}$ & Massarinaceae & $\begin{array}{l}\text { Imperata } \\
\text { cylindrica }\end{array}$ & Thambugala et al. (2017) \\
\hline $\begin{array}{l}\text { *Stagonospora multiseptata } \\
\text { Thambug. \& K.D. Hyde }\end{array}$ & Massarinaceae & $\begin{array}{l}\text { leaves and } \\
\text { stems of } \\
\text { grasses }\end{array}$ & Thambugala et al. (2017) \\
\hline $\begin{array}{l}\text { *Stenocarpella maydis (Berk.) B. } \\
\text { Sutton }\end{array}$ & Diaporthaceae & Zea mays & $\begin{array}{l}\text { Giatgong (1980) as Diplodia } \\
\text { zeae }\end{array}$ \\
\hline $\begin{array}{l}\text { *Sulcosporium thailandicum } \\
\text { Phookamsak \& K.D. Hyde }\end{array}$ & Halotthiaceae & $\begin{array}{l}\text { Axonopus } \\
\text { compressus }\end{array}$ & Ariyawansa et al. (2015b) \\
\hline $\begin{array}{l}\text { *Tetraploa yakushimensis (Kaz. } \\
\text { Tanaka, K. Hiray. \& Hosoya) } \\
\text { Kaz. Tanaka \& K. Hiray. }\end{array}$ & Tetraplosphaeriaceae & $\begin{array}{l}\text { Imperata } \\
\text { cylindrica }\end{array}$ & Phookamsak (2017) \\
\hline $\begin{array}{l}\text { *Ustilaginoidea virens (Cooke) } \\
\text { Takah. }\end{array}$ & Clavicipitaceae & Oryza sativa & Giatgong (1980) \\
\hline $\begin{array}{l}\text { BASIDIOMYCOTA } \\
\text { Anthracocystis andropogonis- } \\
\text { aciculati (Petch) McTaggart \& } \\
\text { R.G. Shivas }\end{array}$ & Ustilaginaceae & $\begin{array}{l}\text { Chrysopogon } \\
\text { aciculatus }\end{array}$ & $\begin{array}{l}\text { Shivas et al. (2007) as } \\
\text { Sporisorium andropogonis- } \\
\text { aciculati }\end{array}$ \\
\hline $\begin{array}{l}\text { *Anthracocystis anthistiriae } \\
\text { (Cobb) McTaggart \& R.G. Shivas }\end{array}$ & Ustilaginaceae & $\begin{array}{l}\text { Themeda } \\
\text { triandra }\end{array}$ & $\begin{array}{l}\text { Shivas et al. (2007) as } \\
\text { Sporisorium anthistiriae }\end{array}$ \\
\hline $\begin{array}{l}\text { Anthracocystis berndtii (Vánky) } \\
\text { McTaggart \& R.G. Shivas }\end{array}$ & Ustilaginaceae & $\begin{array}{l}\text { Schizachyrium } \\
\text { sanguineum }\end{array}$ & $\begin{array}{l}\text { Shivas et al. (2008) as } \\
\text { Sporisorium berndtii, } \\
\text { McTaggart et al. (2012) }\end{array}$ \\
\hline $\begin{array}{l}\text { Anthracocystis holstii (Henn.) } \\
\text { McTaggart \& R.G. Shivas }\end{array}$ & Ustilaginaceae & $\begin{array}{l}\text { Themeda } \\
\text { triandra }\end{array}$ & $\begin{array}{l}\text { Shivas et al. (2007) as } \\
\text { Sporisorium holstii, McTaggart } \\
\text { et al. (2012) }\end{array}$ \\
\hline
\end{tabular}


Table 1 Continued.

\begin{tabular}{|c|c|c|c|}
\hline Species & Family & Host & Reference \\
\hline $\begin{array}{l}\text { Anthracocystis likhitekarajae } \\
\text { (R.G. Shivas, Athip. \& } \\
\text { McTaggart) McTaggart \& R.G. } \\
\text { Shivas }\end{array}$ & Ustilaginaceae & Ischaemum sp. & $\begin{array}{l}\text { Shivas et al. (2008) as } \\
\text { Sporisorium likhitekarajae, } \\
\text { McTaggart et al. } 2012\end{array}$ \\
\hline $\begin{array}{l}\text { Anthracocystis paspali-thunbergii } \\
\text { (Henn.) McTaggart \& R.G. } \\
\text { Shivas }\end{array}$ & Ustilaginaceae & $\begin{array}{l}\text { Paspalum } \\
\text { orbiculare }\end{array}$ & $\begin{array}{l}\text { Shivas et al. (2007) as } \\
\text { Sporisorium paspali- } \\
\text { thunbergii }\end{array}$ \\
\hline $\begin{array}{l}\text { Anthracocystis shivasiorum } \\
\text { (Vánky) McTaggart \& R.G. } \\
\text { Shivas }\end{array}$ & Ustilaginaceae & Eulalia trispicata & $\begin{array}{l}\text { Vánky (2008) as } \\
\text { Sporisorium shivasiorum, } \\
\text { McTaggart et al. (2012) }\end{array}$ \\
\hline $\begin{array}{l}\text { *Anthracocystis trispicatae (R.G. } \\
\text { Shivas, Vánky \& Athip.) } \\
\text { McTaggart \& R.G. Shivas }\end{array}$ & Ustilaginaceae & Eulalia trispicata & $\begin{array}{l}\text { Vánky et al. (2006) as } \\
\text { Sporisorium trispicatae, } \\
\text { Shivas et al. (2007) as } \\
\text { S. trispicatae, McTaggart et } \\
\text { al. (2012), Piątek et al. } \\
\text { (2015) }\end{array}$ \\
\hline $\begin{array}{l}\text { *Conidiosporomyces ayresii } \\
\text { (Berk.) Vánky \& R. Bauer }\end{array}$ & Tilletiaceae & Panicum maximum & Shivas et al. (2007) \\
\hline $\begin{array}{l}\text { *Eballistra oryzae (Syd. \& P. } \\
\text { Syd.) R. Bauer, Begerow, A. } \\
\text { Nagler \& Oberw }\end{array}$ & Entylomataceae & Oryza sativa & $\begin{array}{l}\text { Giatgong (1980) as } \\
\text { Entyloma oryzae }\end{array}$ \\
\hline $\begin{array}{l}\text { *Franzpetrakia microstegii } \\
\text { Thirum. \& Pavgi }\end{array}$ & Ustilaginaceae & $\begin{array}{l}\text { Microstegium } \\
\text { fasciculatum }\end{array}$ & $\begin{array}{l}\text { Shivas et al. (2007), Vánky } \\
\text { (2013) }\end{array}$ \\
\hline $\begin{array}{l}\text { Langdonia clandestina (R.G. } \\
\text { Shivas, Vánky \& Athip.) } \\
\text { McTaggart \& R.G. Shivas }\end{array}$ & Ustilaginaceae & $\begin{array}{l}\text { Aristida balansae } \\
\text { Aristida setacea }\end{array}$ & $\begin{array}{l}\text { McTaggart et al. (2012) } \\
\text { Vánky et al. (2006), Shivas } \\
\text { et al. (2007) as Sporisorium } \\
\text { clandestinum, McTaggart et } \\
\text { al. (2012) }\end{array}$ \\
\hline $\begin{array}{l}\text { *Langdonia inopinata (Vánky) } \\
\text { McTaggart \& R.G. Shivas }\end{array}$ & Ustilaginaceae & Aristida sp. & $\begin{array}{l}\text { Shivas et al. (2007) as } \\
\text { Sporisorium inopinatum, } \\
\text { McTaggart et al. (2012) }\end{array}$ \\
\hline $\begin{array}{l}\text { *Macalpinomyces arundinellae- } \\
\text { setosae R.G. Shivas \& Vánky }\end{array}$ & Ustilaginaceae & Arundinella setosa & Shivas et al. (2007) \\
\hline $\begin{array}{l}\text { Macalpinomyces siamensis R.G. } \\
\text { Shivas, Vánky \& Athip. }\end{array}$ & Ustilaginaceae & Coelorachis striata & $\begin{array}{l}\text { Vánky et al. (2006), Shivas } \\
\text { et al. (2007) }\end{array}$ \\
\hline $\begin{array}{l}\text { *Moesziomyces bullatus (J. } \\
\text { Schröt.) Vánky }\end{array}$ & Ustilaginaceae & $\begin{array}{l}\text { Leersia hexandra, } \\
\text { Polytrias amaura }\end{array}$ & Shivas et al. (2007) \\
\hline $\begin{array}{l}\text { * Ontotelium coronatum Syd. as } \\
\text { Uromyces coronatus }\end{array}$ & Pucciniaceae & Zizania latifolia & $\begin{array}{l}\text { Lorsuwan et al. (1984) as } \\
\text { Uromyces coronatus }\end{array}$ \\
\hline $\begin{array}{l}\text { Puccinia andropogonicola Har. \& } \\
\text { Pat. }\end{array}$ & Pucciniaceae & $\begin{array}{l}\text { Hyparrhenia } \\
\text { bracteata }\end{array}$ & Gjaerum (1988) \\
\hline $\begin{array}{l}\text { *Puccinia cynodontis Lacroix ex } \\
\text { Desm. }\end{array}$ & Pucciniaceae & $\begin{array}{l}\text { Cynodon dactylon } \\
\text { Cynodon sp. }\end{array}$ & $\begin{array}{l}\text { Lenne (1990) } \\
\text { Lorsuwan et al. (1984) }\end{array}$ \\
\hline *Puccinia duthiei Ellis \& Tracy & Pucciniaceae & $\begin{array}{l}\text { Dichanthium } \\
\text { annulatum }\end{array}$ & $\begin{array}{l}\text { Lorsuwan et al. (1984) as } \\
\text { Puccinia duthiae }\end{array}$ \\
\hline *Puccinia graminis Pers & Pucciniaceae & Hordeum vulgare & $\begin{array}{l}\text { Giatgong (1980) as Puccinia } \\
\text { graminis subsp. graminis }\end{array}$ \\
\hline $\begin{array}{l}\text { *Puccinia graminis f.sp. avenae } \\
\text { Erikss. \& Henning }\end{array}$ & Pucciniaceae & Avena sativa & Giatgong (1980) \\
\hline $\begin{array}{l}\text { *Puccinia melanocephala Syd. \& } \\
\text { P. Syd. }\end{array}$ & Pucciniaceae & $\begin{array}{l}\text { Saccharum } \\
\text { officinarum }\end{array}$ & Giatgong (1980) \\
\hline *Puccinia nakanishikii Dietel & Pucciniaceae & $\begin{array}{l}\text { Cymbopogon } \\
\text { citratus }\end{array}$ & Lorsuwan et al. (1984) \\
\hline $\begin{array}{l}\text { Puccinia neyraudiae Syd. \& P. } \\
\text { Syd. }\end{array}$ & Pucciniaceae & $\begin{array}{l}\text { Neyraudia } \\
\text { reynaudiana }\end{array}$ & Lohsomboon et al. (1986) \\
\hline
\end{tabular}


Table 1 Continued.

\begin{tabular}{|c|c|c|c|}
\hline Species & Family & Host & Reference \\
\hline $\begin{array}{l}\text { *Puccinia operta Mundk. \& } \\
\text { Thirum. }\end{array}$ & Pucciniaceae & Coix lacryma-jobi & Lohsomboon et al. (1986) \\
\hline \multirow[t]{2}{*}{ *Puccinia polysora Underw. } & Pucciniaceae & $\begin{array}{l}\text { Brachiaria } \\
\text { distachya }\end{array}$ & Unartngam et al. (2011) \\
\hline & & Zea mays & $\begin{array}{l}\text { Giatgong (1980), Unartngam } \\
\text { et al. (2011) }\end{array}$ \\
\hline \multirow[t]{2}{*}{ *Puccinia purpurea Cooke } & Pucciniaceae & $\begin{array}{l}\text { Sorghum } \\
\text { halepense }\end{array}$ & Giatgong (1980) \\
\hline & & Sorghum vulgare & $\begin{array}{l}\text { Giatgong (1980), Lorsuwan } \\
\text { et al. (1984) }\end{array}$ \\
\hline $\begin{array}{l}\text { *Puccinia recondita Roberge ex } \\
\text { Desm. }\end{array}$ & Pucciniaceae & Triticum aestivum & Lorsuwan et al. (1984) \\
\hline $\begin{array}{l}\text { *Puccinia rubigo-vera f.sp. tritici } \\
\text { Carleton }\end{array}$ & Pucciniaceae & Triticum vulgare & Giatgong (1980) \\
\hline *Puccinia rufipes Dietel & Pucciniaceae & $\begin{array}{l}\text { Imperata } \\
\text { cylindrica }\end{array}$ & $\begin{array}{l}\text { Giatgong (1980), Lorsuwan } \\
\text { et al. (1984) }\end{array}$ \\
\hline *Puccinia sorghi Schwein. & Pucciniaceae & Zea mays & $\begin{array}{l}\text { Giatgong (1980), Lorsuwan } \\
\text { et al. (1984) }\end{array}$ \\
\hline Puccinia sp. & Pucciniaceae & Sorghum sp. & Giatgong (1980) \\
\hline *Puccinia striiformis Westend & Pucciniaceae & Triticum vulgare & $\begin{array}{l}\text { Giatgong (1980) as Puccinia } \\
\text { glumarum }\end{array}$ \\
\hline *Rhizoctonia solani J.G. Kühn & Ceratobasidiaceae & Oryza sativa & $\begin{array}{l}\text { Giatgong }(1980) \text { as } \\
\text { Thanatephorus cucumeris }\end{array}$ \\
\hline *Schizophyllum commune Fr. & Schizophyllaceae & $\begin{array}{l}\text { Saccharum } \\
\text { officinarum }\end{array}$ & Giatgong (1980) \\
\hline $\begin{array}{l}\text { Sporisorium amaurae Vánky \& } \\
\text { C. Vánky }\end{array}$ & Ustilaginaceae & Polytrias amaura & Shivas et al. (2007) \\
\hline $\begin{array}{l}\text { *Sporisorium arthraxonis (Pat.) } \\
\text { L. Guo }\end{array}$ & Ustilaginaceae & Arthraxon hispidus & Shivas et al. (2007) \\
\hline $\begin{array}{l}\text { *Sporisorium cruentum (J.G. } \\
\text { Kühn) Vánky }\end{array}$ & Ustilaginaceae & $\begin{array}{l}\text { Sorghum } \\
\text { propinquum }\end{array}$ & Shivas et al. (2007) \\
\hline $\begin{array}{l}\text { Sporisorium dichanthiicola } \\
\text { (Mundk. \& Thirum.) Vánky }\end{array}$ & Ustilaginaceae & $\begin{array}{l}\text { Dichanthium } \\
\text { caricosum }\end{array}$ & $\begin{array}{l}\text { Shivas et al. (2007) as } \\
\text { Sporisorium dichanthicola }\end{array}$ \\
\hline $\begin{array}{l}\text { *Sporisorium doidgeae (Zundel) } \\
\text { Langdon \& Full. }\end{array}$ & Ustilaginaceae & $\begin{array}{l}\text { Bothriochloa } \\
\text { bladhii }\end{array}$ & Shivas et al. (2007) \\
\hline $\begin{array}{l}\text { *Sporisorium exsertum } \\
\text { (McAlpine) L. Guo }\end{array}$ & Ustilaginaceae & Themeda triandra & Shivas et al. (2008) \\
\hline $\begin{array}{l}\text { Sporisorium ischaemicola (L. } \\
\text { Ling) Vánky }\end{array}$ & Ustilaginaceae & $\begin{array}{l}\text { Ischaemum } \\
\text { indicum }\end{array}$ & Shivas et al. (2007) \\
\hline $\begin{array}{l}\text { *Sporisorium manilense (Syd. \& } \\
\text { P. Syd.) Vánky }\end{array}$ & Ustilaginaceae & Sacciolepis indica & Shivas et al. (2007) \\
\hline $\begin{array}{l}\text { *Sporisorium ophiuri (Henn.) } \\
\text { Vánky }\end{array}$ & Ustilaginaceae & $\begin{array}{l}\text { Rottboellia } \\
\text { cochinchinensis }\end{array}$ & Shivas et al. (2007) \\
\hline $\begin{array}{l}\text { Sporisorium pseudosorghi } \\
\text { Vánky, R.G. Shivas \& Athip. }\end{array}$ & Ustilaginaceae & $\begin{array}{l}\text { Pseudosorghum } \\
\text { fasciculare }\end{array}$ & $\begin{array}{l}\text { Vánky et al. (2006), Shivas } \\
\text { et al. (2007) }\end{array}$ \\
\hline $\begin{array}{l}\text { Sporisorium sacchari (Rabenh.) } \\
\text { Vánky }\end{array}$ & Ustilaginaceae & $\begin{array}{l}\text { Saccharum } \\
\text { arundinaceum }\end{array}$ & Shivas et al. (2007) \\
\hline $\begin{array}{l}\text { *Sporisorium scitamineum (Syd.) } \\
\text { M. Piepenbr., M. Stoll \& Oberw. }\end{array}$ & Ustilaginaceae & $\begin{array}{l}\text { Saccharum sp. } \\
\text { Saccharum } \\
\text { officinarum }\end{array}$ & $\begin{array}{l}\text { Shivas et al. (2007) } \\
\text { Giatgong (1980) as Ustilago } \\
\text { scitaminea, Shivas et al. } \\
(2007) \text { as } U \text {. scitaminea }\end{array}$ \\
\hline $\begin{array}{l}\text { *Sporisorium scitamineum (Syd.) } \\
\text { M. Piepenbr., M. Stoll \& Oberw. }\end{array}$ & Ustilaginaceae & $\begin{array}{l}\text { Saccharum } \\
\text { officinarum }\end{array}$ & $\begin{array}{l}\text { Giatgong (1980) as Ustilago } \\
\text { scitaminea, }\end{array}$ \\
\hline
\end{tabular}


Table 1 Continued.

\begin{tabular}{|c|c|c|c|}
\hline Species & Family & Host & Reference \\
\hline & & Saccharum sp. & $\begin{array}{l}\text { Shivas et al. (2007) as } \\
\text { Ustilago scitaminea } \\
\text { Raboin et al. (2007) as } \\
\text { U. scitaminea }\end{array}$ \\
\hline Sporisorium sp. & Ustilaginaceae & $\begin{array}{l}\text { Ischaemum } \\
\text { indicum }\end{array}$ & Shivas et al. (2007) \\
\hline $\begin{array}{l}\text { *Sporisorium tenue (Syd. \& P. } \\
\text { Syd.) Vánky }\end{array}$ & Ustilaginaceae & $\begin{array}{l}\text { Bothriochloa } \\
\text { bladhii }\end{array}$ & Shivas et al. (2008) \\
\hline \multirow[t]{2}{*}{$\begin{array}{l}\text { *Stollia bothriochloae (L. Ling) } \\
\text { McTaggart \& R.G. Shivas }\end{array}$} & Ustilaginaceae & $\begin{array}{l}\text { Bothriochloa } \\
\text { bladhii }\end{array}$ & $\begin{array}{l}\text { Shivas et al. (2007) as } \\
\text { Macalpinomyces } \\
\text { bothriochloae, McTaggart et } \\
\text { al. (2012) }\end{array}$ \\
\hline & & $\begin{array}{l}\text { Bothriochloa } \\
\text { pertusa }\end{array}$ & $\begin{array}{l}\text { Shivas et al. (2007) as } M \text {. } \\
\text { bothriochloae }\end{array}$ \\
\hline *Stollia bursa (Berk.) McTaggart & Ustilaginaceae & Themeda triandra & McTaggart et al. (2012) \\
\hline \& R.G. Shivas & & Themeda villosa & $\begin{array}{l}\text { Shivas et al. (2008) as } \\
\text { Macalpinomyces bursus, } \\
\text { McTaggart et al. (2012) }\end{array}$ \\
\hline *Stollia ewartii (McAlpine) & Ustilaginaceae & Sorghum nitidum & Shivas et al. (2008) as \\
\hline McTaggart \& R.G. Shivas & & & Macalpinomyces ewartii \\
\hline Thecaphora sp. & Glomosporiaceae & Digitaria sp. & $\begin{array}{l}\text { Giatgong (1980), Lenne } \\
\text { (1990) as Sorosporium sp. }\end{array}$ \\
\hline $\begin{array}{l}\text { *Tilletia barclayana (Bref.) Sacc. } \\
\& \text { P. Syd. }\end{array}$ & Tilletiaceae & Oryza sativa & $\begin{array}{l}\text { Zundel (1953) as Neovossia } \\
\text { horrida, Duran \& Fischer } \\
\text { (1961), Chandrasrikul } \\
\text { (1962) as Tilletia horrida, } \\
\text { Giatgong (1980) as T. } \\
\text { horrida, Shivas et al. (2007) } \\
\text { as T. horrida }\end{array}$ \\
\hline $\begin{array}{l}\text { Tilletia chiangmaiensis R.G. } \\
\text { Shivas, Vánky \& Athip. }\end{array}$ & Tilletiaceae & $\begin{array}{l}\text { Arundinella } \\
\text { bengalensis }\end{array}$ & $\begin{array}{l}\text { Vánky et al. (2006), Shivas } \\
\text { et al. (2007) }\end{array}$ \\
\hline $\begin{array}{l}\text { Tilletia filisora R.G. Shivas, } \\
\text { Vánky \& Athip. }\end{array}$ & Tilletiaceae & $\begin{array}{l}\text { Pennisetum } \\
\text { setosum }\end{array}$ & $\begin{array}{l}\text { Vánky et al. (2006), Shivas } \\
\text { et al. (2007) }\end{array}$ \\
\hline $\begin{array}{l}\text { Tilletia isachnicola R.G. Shivas, } \\
\text { Athip. \& McTaggart }\end{array}$ & Tilletiaceae & Isachne globosa & Shivas et al. (2008) \\
\hline $\begin{array}{l}\text { Tilletia ischaemi Vánky \& N.D. } \\
\text { Sharma }\end{array}$ & Tilletiaceae & $\begin{array}{l}\text { Ischaemum } \\
\text { rugosum }\end{array}$ & Shivas et al. (2007) \\
\hline $\begin{array}{l}\text { Tilletia lageniformis Vánky, C. } \\
\text { Vánky, R.G. Shivas \& Athip. }\end{array}$ & Tilletiaceae & Hyparrhenia rufa & $\begin{array}{l}\text { Vánky et al. (2006), Shivas } \\
\text { et al. (2007) }\end{array}$ \\
\hline $\begin{array}{l}\text { *Tilletia setariae-parviflorae } \\
\text { Vánky \& R.G. Shivas }\end{array}$ & Tilletiaceae & Setaria parviflora & $\begin{array}{l}\text { Vánky (2007), Shivas et al. } \\
\text { (2007) }\end{array}$ \\
\hline Tilletia sp. & Tilletiaceae & Sacciolepis indica & Shivas et al. (2008) \\
\hline $\begin{array}{l}\text { Tilletia thailandica Vánky \& } \\
\text { R.G. Shivas }\end{array}$ & Tilletiaceae & $\begin{array}{l}\text { Eragrostis } \\
\text { amabilis }\end{array}$ & $\begin{array}{l}\text { Vánky (2007), Shivas et al. } \\
\text { (2007) }\end{array}$ \\
\hline *Tilletia vittata (Berk.) Mundk. & Tilletiaceae & $\begin{array}{l}\text { Oplismenus } \\
\text { compositus }\end{array}$ & Shivas et al. (2007) \\
\hline $\begin{array}{l}\text { *Uromyces setariae-italicae } \\
\text { Yoshino }\end{array}$ & Pucciniaceae & $\begin{array}{l}\text { Panicum } \\
\text { purpurascens }\end{array}$ & $\begin{array}{l}\text { Giatgong (1980) as } \\
\text { Uromyces leptodermus, } \\
\text { Lenne (1990) }\end{array}$ \\
\hline *Ustilago coicis Bref. & Ustilaginaceae & $\begin{array}{l}\text { Panicum sp. } \\
\text { Coix lacryma-jobi }\end{array}$ & $\begin{array}{l}\text { Lorsuwan et al. (1984) } \\
\text { Titatarn et al. (1983), } \\
\text { Richardson (1990), Shivas } \\
\text { (2007) }\end{array}$ \\
\hline $\begin{array}{l}\text { *Ustilago cynodontis (Pass.) } \\
\text { Henn. }\end{array}$ & Ustilaginaceae & Cynodon dactylon & Shivas et al. (2007) \\
\hline
\end{tabular}


Table 1 Continued.

\begin{tabular}{|c|c|c|c|}
\hline Species & Family & Host & Reference \\
\hline $\begin{array}{l}\text { Ustilago egenula Syd., P. Syd. \& } \\
\text { E.J. Butler }\end{array}$ & Ustilaginaceae & $\begin{array}{l}\text { Eragrostis } \\
\text { japonica }\end{array}$ & Shivas et al. (2007) \\
\hline *Ustilago esculenta Henn. & Ustilaginaceae & $\begin{array}{l}\text { Zizania aquatica } \\
\text { Zizania latifolia }\end{array}$ & $\begin{array}{l}\text { Zundel (1953) } \\
\text { Shivas et al. (2008) }\end{array}$ \\
\hline Ustilago maydis (DC.) Corda & Ustilaginaceae & Zea mays & $\begin{array}{l}\text { Giatgong (1980), Shivas et } \\
\text { al. (2007) }\end{array}$ \\
\hline *Ustilago neyraudiae Mundk. & Ustilaginaceae & $\begin{array}{l}\text { Neyraudia } \\
\text { reynaudiana }\end{array}$ & Shivas et al. (2007) \\
\hline $\begin{array}{l}\text { *Ustilago nuda (C.N. Jensen) } \\
\text { Kellerm. \& Swingle }\end{array}$ & Ustilaginaceae & Triticum aestivum & $\begin{array}{l}\text { Shivas et al. (2007) as } \\
\text { Ustilago tritici }\end{array}$ \\
\hline $\begin{array}{l}\text { *Ustilago planetella Vánky \& } \\
\text { R.G. Shivas }\end{array}$ & Ustilaginaceae & $\begin{array}{l}\text { Triticum vulgare } \\
\text { Eragrostis } \\
\text { japonica }\end{array}$ & $\begin{array}{l}\text { Giatgong (1980) as } U \text {. tritici } \\
\text { Vánky (2007) }\end{array}$ \\
\hline $\begin{array}{l}\text { Ustilago sclerachnes Wakef. ex } \\
\text { Vánky }\end{array}$ & Ustilaginaceae & $\begin{array}{l}\text { Chionachne } \\
\text { punctata }\end{array}$ & Shivas et al. (2007) \\
\hline Ustilago sp. & Ustilaginaceae & $\begin{array}{l}\text { Brachiaria mutica } \\
\text { Panicum repens }\end{array}$ & $\begin{array}{l}\text { Giatgong (1980) } \\
\text { Lenne (1990), Giatgong } \\
(1980)\end{array}$ \\
\hline $\begin{array}{l}\text { *Ustilago trichophora (Link) } \\
\text { Kunze }\end{array}$ & Ustilaginaceae & $\begin{array}{l}\text { Zoysia japonica } \\
\text { Echinochloa } \\
\text { colonum }\end{array}$ & $\begin{array}{l}\text { Giatgong (1980) } \\
\text { Shivas et al. (2007) }\end{array}$ \\
\hline $\begin{array}{l}\text { *Waitea circinata Warcup \& } \\
\text { P.H.B. Talbot }\end{array}$ & Corticiaceae & Oryza sativa & $\begin{array}{l}\text { Giatgong (1980) as } \\
\text { Rhizoctonia oryzae }\end{array}$ \\
\hline $\begin{array}{l}\text { CHYTRIDIOMYCOTA } \\
\text { * Physoderma maydis (Miyabe) } \\
\text { Miyabe }\end{array}$ & Physodermataceae & $\begin{array}{l}\text { Sorghum vulgare } \\
\text { Zea mays }\end{array}$ & $\begin{array}{l}\text { Giatgong (1980) } \\
\text { Giatgong (1980) as } \\
\text { Physoderma zeae-maydis }\end{array}$ \\
\hline
\end{tabular}

*moledular data available in GenBank. In bold - Type/ species first recorded from this grass host in Thailand.

\section{Conclusions}

This checklist of microfungi on grasses (excluding bambusicolous fungi) found in Thailand, consists of 172 taxa distributed in 24 orders, 46 families and 89 genera. Another 35 taxa remain unidentified (to species level). The species Stagonospora sacchari T.T. Lo \& L. Ling (Phaeosphaeriaceae) recorded on Saccharum officinarum (Castellani \& Germano 1977, Giatgong 1980) and Ustilago phragmitis Hirschh. (Ustilaginaceae) on Phragmites karka (Shivas et al. 2007) are currently listed as invalid names in Index Fungorum and therefore are not included in this checklist. The most commonly recorded genera are Puccinia, Sporisorium, Tilletia and Ustilago (commonly known as the rusts and smuts), followed by the genera Bipolaris/Cochliobolus, Curvularia, Cercospora, Colletotrichum and Fusarium.

\section{Acknowledgements}

Ishani D. Goonasekara expresses her gratitude to the Mushroom Research Foundation, Chiang Mai, Thailand for financial assistance.

\section{References}

Ariyawansa HA, Hyde KD, Jayasiri SC, Buyck B et al. 2015a - Fungal diversity notes 111-252taxonomic and phylogenetic contributions to fungal taxa. Fungal Diversity 75, 27-274.

Ariyawansa HA, Thambugala KM, Manamgoda DS, Jayawardena R et al. 2015b - Towards a natural classification and backbone tree for Pleosporaceae. Fungal Diversity 71, 85-139. 
Bakhshi M, Arzanlou M, Babai-Ahari A, Groenewald JZ et al. 2015 - Application of the consolidated species concept to Cercospora spp. from Iran. Persoonia 34, 65-86.

Bandyopadhyay R, Frederickson DE, McLaren NW, Odvody GN et al. 1998 - Ergot: a new disease threat to sorghum in the Americas and Australia. Plant Disease 82, 356-367.

Bhilabutra W, McKenzie EHC, Hyde KD, Lumyong S. 2010 - Fungi on the grasses, Thysanolaena latifolia and Saccharum spontaneum, in northern Thailand. Mycosphere, 1(4), 301-314.

Braun U, Crous PW, Nakashima C. 2015 - Cercosporoid fungi (Mycosphaerellaceae) 3. Species on monocots (Poaceae, true grasses). IMA Fungus 6, 25-97.

Brook PJ. 1963 - Ecology of the fungus Pithomyces chartarum (Berk. \& Curt.) MB Ellis in pasture in relation to facial eczema disease of sheep. New Zealand Journal of Agricultural Research $6,147-228$.

Castellani E, Germano G 1977 - Le Stagonosporaegraminicole. Annali Facolti di Scienze Agriculturae Universitatis Torino 10, 1-135.

Chandrasrikul A 1962 - A preliminary host list of plant diseases in Thailand. Thailand Deptarment of Agriculture, Technical Bulletin, 6, 23pp.

Clay K. 1988 - Fungal endophytes of grasses: a defensive mutualism between plants and fungi. Ecology 69, 10-16.

Clay K, Marks S, Cheplick GP. 1993 - Effects of insect herbivory and fungal endophyte infection on competitive interactions among grasses. Ecology 74, 1767-1777.

Crouch JA. 2014 - Colletotrichum caudatum s.l. is a species complex. IMA Fungus 5, 17-30.

Crous PW, Wingfield MJ, Guarro J, Cheewangkoon R et al. 2013 - Fungal Planet description sheets: 154-213. Persoonia 31, 188-296.

Dai DQ, Phookamsak R, Wijayawardene NN, Li WJ et al. 2017 - Bambusicolous fungi. Fungal Diversity $82,1-105$.

Damm U, O'Connell RJ, Groenewald JZ, Crous PW. 2014 - The Colletotrichum destructivum species complex-hemibiotrophic pathogens of forage and field crops. Studies in Mycology $79,49-84$.

Diao YZ, Zhang C, Liu F, Wang WZ et al. 2017 - Colletotrichum species causing anthracnose disease of chili in China. Persoonia 38, 20-37.

Duran R, Fischer GW. 1961 - The Genus Tilletia. Washington State University, 138 pp.

Encyclopedia of Life. 2018 - http://eol.org (last accessed on $11^{\text {th }}$ July 2018)

Farr DF, Rossman AY. 2018 - Fungal Databases, U.S. National Fungus Collections, ARS, USDA. Retrieved August 5, 2018, from https://nt.ars-grin.gov/fungaldatabases/

Food and Agriculture Organization of the United Nations (FAO) - http://agris.fao.org (last accessed on $11^{\text {th }}$ July 2018)

Giatgong P. 1980 - Host Index of Plant Diseases in Thailand. Second Edition. Mycology Branch, Plant Pathology and Microbiology Division, Department of Agriculture and Cooperatives, Bangkok, Thailand, 118. (44271)

Gibson DJ. 2009 - Grasses and grassland ecology. Oxford University Press.

Gjaerum HB. 1988 - Rust fungi (Uredinales) on the genus Hyparrhenia (Poaceae). Mycotaxon 32, $143-160$.

Hyde KD, Jones EBG, Liu JK, Ariyawansa H et al. 2013 - Families of Dothideomycetes. Fungal Diversity 63, 1-313.

Hyde KD, Hongsanan S, Jeewon R, Bhat DJ et al. 2016 - Fungal diversity notes 367-491: taxonomic and phylogenetic contributions to fungal taxa. Fungal Diversity 80(1), 1-270

Hyde KD, Chaiwan N, Norphanphoun C, Boonmee S et al. 2018 - Mycosphere notes 169-224. Mycosphere 9(2), 271-430, Doi 10.5943/mycosphere/9/2/8

Index Fungorum. 2018 - http://www.indexfungorum.org/Names/Names.asp

Jeon SJ, Nguyen TT, Lee HB. 2015 - Phylogenetic status of an unrecorded species of Curvularia, C. spicifera, based on current classification system of Curvularia and Bipolaris group using multi loci. Mycobiology 43(3), 210-7.

Kellogg EA. 2001 - Evolutionary history of the grasses. Plant physiology 125, 1198-1205. 
Khemmuk W, Shivas RG, Henry RJ, Geering ADW. 2016 - Fungi associated with foliar diseases of wild and cultivated rice (Oryza spp.) in northern Queensland. Australasian Plant Pathology 45, 297-308.

Lamprecht SC, Crous PW, Groenewald JZ, Tewoldemedhin YT, Marasas WF. 2011 Diaporthaceae associated with root and crown rot of maize. IMA fungus 2, 13-24.

Lenne JM. 1990 - World List of Fungal Diseases of Tropical Pasture Species. Phytopathology Papers 31, 1-162.

Li GJ, Hyde KD, Zhao RN, Hongsanan S et al. 2016 - Fungal diversity notes 253-366: taxonomic and phylogenetic contributions to fungal taxa. Fungal Diversity 78, 1-237.

Liu F, Weir BS, Damm U, Crous PW et al. 2015a - Unravelling Colletotrichum species associated with Camellia: employing ApMat and GS loci to resolve species in the C. gloeosporioides complex. Persoonia 35, 63-86.

Liu JK, Hyde KD, Jones EBG, Ariyawansa HA et al. 2015b - Fungal diversity notes 1-110: taxonomic and phylogenetic contributions to fungal species. Fungal Diversity 72, 1-197.

Lohsomboon P, Manoch L, Visarathanonth N, Kakishima M et al. 1986 - Materials for the rust flora in Thailand II. Transactions of the Mycological Society of Japan 27, 271-281.

Lorsuwan C, Tontyaporn S, Visarathanonth N, Manoch L et al. 1984 - Materials for the rust flora in Thailand I. Transactions of the Mycological Society of Japan 25, 57-65.

Maharachchikumbura SSN, Hyde KD, Jones EBG, McKenzie EHC et al. 2016 - Families of Sordariomycetes. Fungal Diversity 79, 1-317.

Manamgoda DS, Cai L, Bahkali AH, Chukeatirote E et al. 2011 - Cochliobolus: an overview and current status of species. Fungal Diversity 51, 3-42.

Manamgoda DS, Cai L, McKenzie EHC, Crous PW et al. 2012a - A phylogenetic and taxonomic re-evaluation of the Bipolaris - Cochliobolus - Curvularia Complex. Fungal Diversity 56, 131-144.

Manamgoda DS, Cai L, McKenzie EHC, Chukeatirote E et al. 2012b - Two new Curvularia species from northern Thailand. Sydowia 64, 255-266.

Manamgoda DS, Udayanga D, Cai L, Chukeatirote E et al. 2013 - Endophytic Colletotrichum from tropical grasses with a new species Colletotrichum endophytica. Fungal Diversity 61, 107115 .

Manamgoda DS, Rossman AY, Castlebury LA, Crous PW et al. 2014 - The genus Bipolaris. Studies in Mycology 79, 221-88.

Manamgoda DS, Rossman AY, Castlebury LA, Chukeatirote E et al. 2015 - A taxonomic and phylogenetic re-appraisal of the genus Curvularia (Pleosporaceae): human and plant pathogens. Phytotaxa 212, 175-198.

Marin-Felix Y, Senwanna C, Cheewangkoon R, Crous PW. 2017 - New species and records of Bipolaris and Curvularia from Thailand. Mycosphere 8(9), 1556-1574.

McTaggart AR, Shivas RG, Geering ADW, Vánky K et al. 2012 - Taxonomic revision of Ustilago, Sporisorium and Macalpinomyces. Persoonia 29, 116-132.

Munir M, Amsden B, Dixon E, Vaillancourt L et al. 2016 - Characterization of Colletotrichum species causing bitter rot of apple in Kentucky orchards. Plant Disease 100(11), 2194-2203.

Nag Raj TR. 1993 - Coelomycetous anamorphs with appendage-bearing conidia. Mycologue Publications, Waterloo, Ontario, 1101pp.

Ou SH. 1985 - Rice Diseases. Second Edition. Commonwealth Mycological Institute, Kew, Surrey, England, $380 \mathrm{pp}$.

Pazoutova S, Kolarik M. Kolinska R. 2004 - Pleomorphic conidiation in Claviceps. Mycological Research 108, 126-135.

Pazoutova S, Frederickson DE. 2005 - Genetic diversity of Claviceps africana on sorghum and Hyparrhenia. Plant Pathology 54, 749-763.

Phengsintham P, Chukeatirote E, McKenzie EHC, Moslem MA et al. 2012 - Fourteen new records of cercosporoids from Thailand. Maejo International Journal of Science and Technology 6, 47-61. 
Phengsintham P, Braun U, McKenzie EHC, Chukeatirote E et al. 2013 - Monograph of Cercosporoid fungi from Thailand. Plant Pathology \& Quarantine 3, 67-138.

Phookamsak R. 2017 - Taxonomy and Phylogeny of selected Dothideomycetes on Monocots. PhD Thesis, Mae Fah Luang University, Chiang Rai, Thailand.

Phookamsak R, Liu JK, McKenzie EHC, Manamgoda DS et al. 2014a - Revision of Phaeosphaeriaceae. Fungal Diversity 68, 159-238.

Phookamsak R, Liu JK, Manamgoda DS, Chukeatirote et al. 2014b - The sexual state of Setophoma. Phytotaxa 176(1), 260-269.

Phookamsak R, Norphanphoun C, Tanaka K, Dai DQ et al. 2015 - Towards a natural classification of Astrosphaeriella-like species; introducing Astrosphaeriellaceae and Pseudoastrosphaeriellaceae fam. nov. and Astrosphaeriellopsis, gen. nov. Fungal Diversity 74, 143-197.

Piątek M, Lutz M, Yorou NS. 2015 - A molecular phylogenetic framework for Anthracocystis (Ustilaginales), including five new combinations (inter alia for the asexual Pseudozyma flocculosa), and description of Anthracocystis grodzinskae sp. nov. Mycological Progress 14(10), 88pp.

Prasad V, Strömberg CAE, Leaché AD, Samant B et al. 2011 - Late Cretaceous origin of the rice tribe provides evidence for early diversification in Poaceae. Nature Communications 2, 480.

Punithalingam E. 1975 - Some new species and combinations in Phomopsis. Transactions of the British Mycological Society 64, 427-435.

Punithalingam E, Sharma ND. 1979 - New microfungi from south east Asia. Nova Hedwigia 31, 881-897.

Purahong W, Hyde KD. 2011 - Effects of fungal endophytes on grass and non-grass litter decomposition rates. Fungal Diversity 47(1), 1-7.

Quaedvlieg W, Verkley GJM, Shin HD, Barreto RW et al. 2013 - Sizing up Septoria. Studies in Mycology 75, 307-390.

Raboin LM, Selvi A, Oliveira KM, Paulet F et al. 2007 - Evidence for the dispersal of a unique lineage from Asia to America and Africa in the sugarcane fungal pathogen Ustilago scitaminea. Fungal Genetics and Biology 44, 64-76.

Richardson MJ. 1990 - An Annotated List of Seed-Borne Diseases. Fourth Edition. International Seed Testing Association, Zurich.

Ryley MJ. 2006 - Notes on the type, synonyms, and other specimens of the balansioid fungus, Nigrocornus scleroticus. Mycotaxon 95, 97-112.

Saikkonen K, Helander M, Faeth SH, Schulthess F, Wilson D. 1999 - Endophyte-grass-herbivore interactions: the case of Neotyphodium endophytes in Arizona fescue populations. Oecologia 121, 411-420.

Sharma G, Shenoy BD. 2014 - Colletotrichum fructicola and C. siamense are involved in chili anthracnose in India. Archives of Phytopathology and Plant Protection 47(10), 1179-1194.

Sharp D, Simon BK. 2002 - AusGrass: Grasses of Australia. Australian Biological Resources, Canberra and Environmental Protection Agency, Queensland.

Sherriff C, Whelan MJ, Arnold GM, Bailey JA. 1995 - rDNA sequence analysis confirms the distinction between Colletotrichum graminicola and C. sublineolum. Mycological Research 99, 475-478.

Shivas RG, Athipunyakom P, Likhitekaraj S, Butranu W et al. 2007 - An annotated checklist of smut fungi (Ustilaginomycetes) from Thailand. Australasian Plant Pathology 36, 376-382.

Shivas RG, Athipunyakom P, McTaggart AR. 2008 - New records of smut fungi (Ustilaginomycetes) from Thailand, incluing two new species, Sporisorium likhitekarahjae and Tilletia isachneicola. Mycologia Balcanica 5, 103-107.

Sivanesan A.1987 - Graminicolous species of Bipolaris, Curvularia, Drechslera, Exserohilum and their teleomorphs. Mycological Papers 158, 1-261.

Smut fungi of Thailand. - http://collections.daff.qld.gov.au/web/key/thaismutfungi/Media/ Html/host.html\#A (last accessed $10^{\text {th }}$ August 2018) 
Somrithipol S, Jones EBG. 2003 - Pseudoacrodictys dimorphospora sp. nov., a new graminicolous hyphomycete from Thailand. Sydowia 55(6), 365-371.

Tamakaew N, Maharachchikumbura SSN, Hyde KD, Cheewangkoon R. 2017 - Tar spot fungi in Thailand. Mycosphere 8(8), 1054-1058.

Tan YP, Crous PW, Shivas RG. 2016 - Eight novel Bipolaris species identified from John L. Alcorn's collections at the Queensland Plant Pathology Herbarium (BRIP). Mycological Progress 15, 1203-1214.

Thambugala KM, Wanasinghe DN, Phillips AJL, Camporesi E et al. 2017 - Mycosphere notes 1-50: Grass (Poaceae) inhabiting. Mycosphere 8, 697-796, Doi 10.5943/mycosphere/8/4/13

The Plant List. 2018 - http://www.theplantlist.org (last accessed on $10^{\text {th }}$ July 2018)

Titatarn S, Chiengkul A, Unchalisangkas D, Chamkrachang W et al. 1983 - Occurrence of Ustilago coicis on Coix lachryma-jobi in Thailand. Plant Disease 67, 434-435.

Trakunyingcharoen T, Lombard L, Groenewald JZ, Cheewangkoon R et al. 2014 - Mycoparasitic species of Sphaerellopsis, and allied lichenicolous and other genera. IMA Fungus 5(2), 391414.

Udayanga D, Liu X, McKenzie EHC, Chukeatirote E et al. 2011 - The genus Phomopsis: biology, applications, species concepts and names of common phytopathogens. Fungal Diversity 50, 189-225.

Unartngam J, Janruang P, To-anan C. 2011 - Genetic diversity of Puccinia polysora in Thailand based on Inter Simple Sequence Repeat (ISSR) markers analysis. Journal of Agricultural Technology 7, 1125-1137.

Vánky K. 2007 - Taxonomic studies on Ustilaginomycetes - 27. Mycotaxon 99, 1-70.

Vánky K. 2008 - Taxonomic studies of Ustilaginomycetes -28. Mycotaxon 106, 133-178.

Vánky K. 2012 - Smut fungi of the world. American Phytopathological Society.

Vánky, K. 2013 - Illustrated genera of smut fungi. APS Press, St. Paul, Minnesota, 288 pp.

Vánky K, Shivas RG, Athipunyakom P. 2006 - New smut fungi (Ustilaginomycetes) from Thailand. Mycologia Balcanica 3, 107-118.

Watson L. 1990 - The grass family, Poaceae. Reproductive versatility in the grasses 1-31.

Wheeler DJ, Jacobs SW, Norton BE. 1990 - Grasses of New South Wales. University of New England.

Wijayawardene NN, Hyde KD, Wanasinghe DN, Papizadeh M et al. 2016 - Taxonomy and phylogeny of dematiaceous coelomycetes. Fungal Diversity 77, 1-316.

Wijayawardene NN, Hyde KD, Lumbsch HT, Liu JK et al. 2018 - Outline of Ascomycota: 2017. Fungal Diversity 88, 167-263.

Wikee S, Lombard L, Crous PW, Nakashima C et al. 2013a - Phyllosticta capitalensis, a widespread endophyte of plants. Fungal Diversity 60, 91-105.

Wikee S, Lombard L, Nakashima C, Motohashi K et al. 2013b - A phylogenetic re-evaluation of Phyllosticta (Botryosphaeriales). Studies in Mycology 76, 1-29.

Wong MK, Hyde KD. 2001 - Diversity of fungi on six species of Gramineae and one species of Cyperaceae in Hong Kong. Mycological Research 105, 1485-1491.

Xiao ZL, Hyde KD, Zhang JZ. 2015 - Synonymy of two species of Bipolaris from aquatic crops of Poaceae. Mycotaxon 130(1), 131-143.

Zhang W, Liu J, Huo PH, Zhang T et al. 2017 - Characterization and pathogenicity of Bipolaris peregianensis: the causal organism for leaf spot of hybrid bermudagrass in China. European Journal of Plant Pathology 148(3), 551-555.

Zhang W, Liu JX, Huo PH, Huang Z. 2018 - Curvularia malina causes a foliar disease on hybrid Bermuda grass in China. European Journal of Plant Pathology 151(2), 557-562.

Zundel GL. 1953 - The Ustilaginales of the World. Contributions from the Department of Botany Pennsylvania State College, School of Agriculture 176, 1-410. 